\title{
Potential for SARS-CoV-2 virus exposure during gynaecological procedures
}

Dr Claire Shannon-Lowe ${ }^{1}$, Dr Heather M Long ${ }^{1}$, Prof Sudha Sundar ${ }^{2 *}$, Dr Graham S Taylor ${ }^{1 *}$

${ }^{1}$ Institute of Immunology and Immunotherapy, University of Birmingham, Vincent Drive, Birmingham, B15 2TT. United Kingdom

2 Institute of Cancer and Genomic Sciences, University of Birmingham, Vincent Drive, Birmingham, B15 2TT. United Kingdom

* correspondence S.S.Sundar@bham.ac.uk; G.S.Taylor@bham.ac.uk

The potential for transmission of the SARS-CoV-2 virus during minimally invasive gynaecological procedures, such as hysteroscopy or laparoscopy, on the reproductive tract of patients with COVID-19 is not known. We examined existing data for prevalence of virus in the reproductive tract and other bodily fluids. A Medline search, $26^{\text {th }}$ March 2020 did not find any studies directly measuring the presence of infectious viral particles. Several reports have used different PCR molecular assays, likely varying in sensitivity, to detect viral RNA which may indicate the presence of infectious virus. These assays show viral RNA is present in a range of bodily specimens from COVID-19 patients including upper and lower respiratory tract samples, faeces and blood ${ }^{1-4}$.

The presence of viral RNA in blood raises the possibility of blood-borne transmission via accidental inoculation or aerosols generated during clinical procedures. Only a minority of COVID-19 patients have detectable viral RNAaemia. The frequency of viral RNA-positive blood samples in symptomatic people is reported to be $1 \%{ }^{1}, 8 \%{ }^{2}, 11.5 \%^{3}$ or $15 \%{ }^{4}$. COVID19 patients with severe symptoms have higher viral loads in the nose and throat than patients with mild symptoms ${ }^{5}$. It is not known if virus levels in the blood are similarly linked to disease severity, but levels of the related SARS-CoV-1 virus have previously been shown to be higher in the blood of patients with severe disease ${ }^{6}$. The different frequencies of viral RNA positive blood samples reported for COVID-19 patients could therefore be due to differences in disease severity of the different patient cohorts studied or differences in sensitivity of the PCR assays employed.

It is currently not known if people asymptomatically infected with SARS-CoV-2 have viral RNA in their blood. However, levels of virus in the nose and throat of an asymptomatically infected individual were similar to those measured in symptomatic patients ${ }^{7}$. Regarding the relative levels of viral RNA in different sample types, two of the above studies applied single PCR assays to multiple sample types allowing this question to be addressed. Both studies reported viral RNA levels in blood are always lower than those measured in respiratory samples ${ }^{1,3}$. Using the number of PCR cycles required to generate an amplification product to estimate viral RNA levels, the amount of viral RNA in blood was eight times lower than those measured in bronchoalveaolar lavage fluid or sputum and 1024 times lower than measured using nasal swabs ${ }^{1}$; nasal swabs being the sample type that usually yields the highest levels of virus ${ }^{1,7}$. Absolute quantification of viral RNA in different secretions is, however, required to confirm these differences. One study also measured viral RNA levels for 20 patients of whom each provided two to six different samples simultaneously ${ }^{1}$. These data again showed viral RNA levels in the blood were low compared to levels in respiratory tract specimens. Based on current knowledge of SARSCoV-2 presence in blood, the European Centre for Disease Control and Prevention recommended on the $20^{\text {th }}$ March 2020 that donation of substances of human origin (such as 
blood) can continue providing donors fulfil certain criteria ${ }^{8}$. These include not having a current diagnosis of COVID-19, at least 14 days have passed since a COVID-19 diagnosis (and tests for the virus are negative) and the donor has not had contact with a COVID-19 case in the 14 days prior to donation.

Three studies have examined if SARS-CoV-2 can be transmitted vertically to newborns. Evidence of transmission could indicate viral exposure to the infant during gestation or from the reproductive tract if delivered vaginally. Chen and colleagues report that all nine children delivered by caesarean section from women with COVID-19 pneumonia were negative for SARS-CoV-2 RNA ${ }^{9}$. Amniotic fluid, cord blood and breast milk samples were also negative. A separate study of four women with COVID-19, one of whom had a vaginal delivery, similarly failed to detect virus in the infants ${ }^{10}$. A third study of two women with COVID-19, both of whom gave birth by caesarean section, also did not detect virus in the infants ${ }^{11}$. Both women tested positive for the virus in respiratory samples taken before and after birth, but no virus was detected in their amniotic fluid, cord blood, breast milk or vaginal swabs.

\section{Conclusions}

The question whether SARS-CoV-2 could be transmitted during gynaecological procedures has not been directly addressed. Viral RNA can be detected in a range of samples types, including blood. However, viral RNA is present in the blood of only a minority of COVID-19 patients and levels are lower than in respiratory secretions. The prevalence and level of viral RNA in the blood may be lower in patients with less severe disease. It is not known if viral RNA is present in the blood of asymptomatically infected individuals. Current guidance on blood donation is that people may donate blood during the pandemic providing they lack active disease and have not had recent contact with a COVID-19 case. Potential risks from virus in the alimentary canal and shed in faeces should be considered. Analysis of newborn infants did not find evidence of vertical transmission from their mothers and no viral RNA was detected from vaginal swabs taken from two women with active COVID-19 at the time of birth.

\section{Interpretation for clinical practice}

We examined existing data for prevalence of SARS-CoV-2 RNA in the reproductive tract and other bodily fluids. Currently there are no data to support the presence of the SARS-CoV-2 virus in the reproductive tract, even in patients with COVID-19. However, this conclusion is based on a very small number of patients tested and further studies are needed.

Given the evidence we have to date, a reasonable assumption would be that that the risk of transmission from surgery on the reproductive tract in a patient who is asymptomatic (no COVID-19 symptoms in previous 7 days) is likely to be low. The possibility of operating on asymptomatically-infected individuals could be further reduced by avoiding surgery on people who have been in contact with a known COVID-19 case in the previous 14 days. In symptomatic patients and patients who are known COVID-19 positive, the risk vs benefit of surgery will need to be evaluated on an individualised basis. Safe surgical practice and national infection protocols should be followed in all. 


\section{References}

1. Wang W, Xu Y, Gao R, Lu R, Han K, Wu G et al. et al. Detection of SARS-CoV-2 in Different Types of Clinical Specimens. Journal of the American Medical Association; 2020. https://doi.org/10.1001/jama.2020.3786.

2. Young BE, Ong SWX, Kalimuddin S, Low JG, Tan SY, Loh J et al. Epidemiologic Features and Clinical Course of Patients Infected With SARS-CoV-2 in Singapore. Journal of the American Medical Association; 2020. https://doi.org/10.1001/jama.2020.3204.

3. Chan JF, Yip CC, To KK, Tang TH, Wong SC, Leung KH et al. Improved molecular diagnosis of COVID-19 by the novel, highly sensitive and specific COVID-19-RdRp/Hel real-time reverse transcription-polymerase chain reaction assay validated in vitro and with clinical specimens. Journal of Clinical Microbiology; 2020. https://doi.org/ 10.1128/JCM.00310-20

4. Huang C, Wang Y, Li X, Ren L, Zhao J, Hu Y et al. Clinical features of patients infected with 2019 novel coronavirus in Wuhan, China. Lancet; 2020. https://doi.org/ 10.1016/S0140-6736(20)30183-5

5. Liu Y, Yan LM, Wan L, Xiang TX, Le A, Liu JM et al. Viral dynamics in mild and severe cases of COVID-19. Lancet Infectious Diseases; 2020 https://doi.org/ 10.1016/S14733099(20)30232-2.

6. Hung IFN, Cheng VCC, Wu AKL, Tang BSF, Chan KF et al. Viral loads in clinical specimens and SARS manifestations. Emerging Infectious Disease; 2004. https://doi.org/10.3201/eid1009.040058.

7. Zou L, Ruan F, Huang $M$, Liang L, Huang $H$, Hong Zet al. SARS-CoV-2 Viral Load in Upper Respiratory Specimens of Infected Patients. New England Journal of Medicine; 2020. https://doi.org/10.1056/NEJMc2001737

8. European Centre for Disease Prevention and Control. Coronavirus disease 2019 (COVID-19) and supply of substances of human origin in the EU/EEA guidance document. https://www.ecdc.europa.eu/sites/default/files/documents/covid-19supply-substances-human-origin.pdf [accessed 26th March 2020)

9. Chen H, Guo J, Wang C, Luo F, Yu X, Zhang W et al. Clinical characteristics and intrauterine vertical transmission potential of COVID-19 infection in nine pregnant women: a retrospective review of medical records. Lancet; 2020. https://doi.org/ 10.1016/S0140-6736(20)30360-3.

10. Chen Y, Peng H, Wang L, Zhao Y, Zeng L, Gao H et al. Infants born to mothers with a new coronavirus (COVID-19). Frontiers in Pediatrics; 2020. https://doi.org/10.3389/fped.2020.00104.

11. Fan C, Lei D, Fang C, Li C, Wang M, Liu Y et al. Perinatal Transmission of COVID-19 Associated SARS-CoV-2: Should We Worry? Clinical Infectious Diseases; 2020. https://doi.org/10.1093/cid/ciaa226. 